\title{
A Bicriteria-Optimization-Approach-Based Dimensionality-Reduction Model for the Color Display of Hyperspectral Images

\author{
Max Mignotte
}

\begin{abstract}
This paper proposes a new nonlinear dimensionalityreduction model based on a bicriteria global optimization approach for the color display of hyperspectral images. The proposed fusion model is derived from two well-known and contradictory criteria of good visualization, which are useful in any multidimensional imagery color display, namely, accuracy, with the preservation of spectral distance criterion, and contrast, guaranteeing that colors are well distinguished or concretely allowing the good separability of each observed existing material in the final visualized color image. An internal parameter allows our algorithm to express the contribution or the importance of these two criteria for a specific application. In this framework, which also can be viewed as a classical Bayesian optimization strategy involving a tradeoff between fidelity to the unreduced (raw) spectral data and the expected highly contrasted resulting mapping, we will show that a hybrid optimization strategy, combining a global and deterministic optimization procedure and a local stochastic search using the Metropolis criterion, can be exploited to efficiently minimize the complex nonlinear objective cost function related to our model. The experiments reported in this paper demonstrate that the proposed model, taking into account these two criteria of good visualization, makes easier and more reliable the interpretation and quick overview of such multidimensional hyperspectral images.
\end{abstract}

Index Terms-Bayesian model, color display model, complete graph, dimensionality-reduction model, FastMap optimization, local exploration search, low dimensional embedding, Metropolis algorithm, multicriteria optimization, multidimensional hyperspectral imagery, nonstationary Markov random field model, stress function.

\section{INTRODUCTION}

$\mathbf{N}$ OWADAYS, hyperspectral imaging sensors have revolutionalized the field of remote sensing by combining the science of spectroscopy with that of imaging. By its ability to collect the same image scene on many bands of the light spectrum, the resulting 3-D image or hyperspectral data cube makes it possible to derive, for each pixel, a continuous and unique reflectance spectrum which is of great importance, for example, in geology and geophysics for identifying the Earth's surface materials such as particular mineral deposits or types

Manuscript received October 6, 2010; revised February 7, 2011, May 4, 2011, and June 14, 2011; accepted June 19, 2011. Date of publication August 8, 2011; date of current version January 20, 2012. This work was supported by a Natural Sciences and Engineering Research Council of Canada individual research grant.

The author is with the Département d'Informatique et de Recherche Opérationnelle, Faculté des Arts et des Sciences, Université de Montréal, Montréal, QC H3C 3J7, Canada (e-mail: mignotte@iro.umontreal.ca).

Color versions of one or more of the figures in this paper are available online at http://ieeexplore.ieee.org.

Digital Object Identifier 10.1109/TGRS.2011.2160646 of vegetation. In practical applications, it may be interesting if this huge amount of high-dimensional spectral data information is reduced to three dimensions. This allows us to quickly display this data cube into an informative color image (with red $(\mathrm{R})$, green $(\mathrm{G})$, and blue $(\mathrm{B})$ channels) and to provide a quick overview of existing materials and their distribution in the image scene for further analysis. Obviously, such a threecolor channel display results in significant loss of information of the spectral content, and consequently, this dimensionalityreduction-based visualization step should be done according to the most appropriate (possibly statistical) criterion.

To this end, dimensionality-reduction methods based on linear-projection methods such as Independent Component Analysis (ICA) [1], Principal Component Analysis (PCA) [2] (with its numerous variants [3]-[5]), and Projection Pursuit (PP) [6] have commonly been proposed in the literature to obtain the first three principal R, G, and B image components to be finally visualized. However, since ICA, PCA, and PP are linear-projection methods, all three assume that the underlying data manifold is linear, which is not necessarily true in the case of hyperspectral images [7]. Moreover, it must be noted that the use of ICA (or, more generally, the use of a mutual information criterion [8]) is based on the assumption of mutually independent sources, which is not really the case of hyperspectral data [9]. Another interesting linear-projection-based color visualization approach is proposed in [10] and further explored and discussed in [11]. In their approach, the three displayed channels are simply estimated by linear integrations of the original hyperspectral image weighted by three different and fixed spectral weighting envelopes (corresponding to $\mathrm{R}$, $\mathrm{G}$, and $\mathrm{B}$ channels), just like the human photopic (daylight) vision works. However, since the spectral weighting envelopes are fixed, there is no adaptation to specific image information. It is also worth mentioning the linear-projection-based fusion strategy proposed in [12], which tries to define the fused image as a weighted sum of individual components, where the weight reflects the locally dominating features of a pixel in a given band. Multiresolution [13] and wavelet decompositions [14] have also been reported. In these methods, selection and fusion rules determine which spectral band is most relevant in a neighborhood of a given pixel and how the features of this selected band are then incorporated into the fused image. These methods implicitly assume that there is only one dominant band at each pixel which is not true in multispectral imagery due to the existing large interband correlation. Let us finally mention the low-complexity color display approach (suitable 
for hardware implementation) proposed in [15] which exploits the 1-b transform of hyperspectral image bands for selecting three suitable bands for the RGB display, the multiscale representation proposed in [16], the multivariate visualization technique proposed in [17] (and recently further improved in [18]) which uses double-color layers displaying simultaneously on the first layer (referred to as the background layer) the distribution of materials existing in the image scene and, in the second layer (detail layer), their respective composition (i.e., the so-called end-member materials) at the subpixel level, or finally, the dimensionality-reduction method proposed in [19] decorrelating and compressing the spatial and spectral dimensions simultaneously.

In order to overcome the main limitation of these linearprojection methods which mainly do not consider the nonlinear characteristics of the hyperspectral data [7], an alternative idea consists of exploiting a nonlinear dimensionality-reduction method (into three bands) such as the locally linear embedding (LLE) method [20]. Nevertheless, this technique is very time consuming compared with linear-projection methods. In contrast to these aforementioned linear or nonlinear dimensionality-reduction methods, also called featureextraction techniques [21], which search to find the transformation from a higher dimension to a lower dimensional feature space with most of the desired information content preserved, dimensionality-reduction schemes, based on a feature-selection strategy, have also been proposed in hyperspectral imagery [21] in order to clearly identify the variables that do not contribute to class separability and which should be neglected. Finally, a recent paper in which different color display techniques are reviewed, compared, and evaluated is proposed in [22].

As we have noted earlier, this dimensionality-reductionbased visualization (or fusion) step should be done according to the most appropriate criterion. In hyperspectral imagery, the most appropriate goal of any good visualization should be mainly based on the now well-known preservation of spectral distance criterion [10], [23], [24] which measures the agreement between the distance of spectrums associated to each pair of pixels and their perceptual color distance in the final fused image to be displayed. This criterion allows us to provide a meaningful color visualization map which is faithful to the unreduced (raw) spectral data. Another important criterion of good visualization is the contrast or the separability of features [23] (i.e., the separability of each observed existing material or class) in the final visualized color image. This allows us to display, for example, the different scene elements as distinctively as possible (eventually for further analysis). In fact, the preservation of spectral distance criterion alone does not guarantee that colors can be well distinguished; the resulting (color) image may be of low contrast (i.e., flat and too dark or too bright) and can result, for an observer, in substantial loss of visual information even if the main preservation of spectral distance criterion indicates that there is very little loss of information in the dimensionality-reduction method which is used. Nevertheless, these two aforementioned criteria of good visualization are somewhat contradictory (as pointed out in [23]) since a high contrast in the final image can be obtained at the cost of numerous saturated pixel values (e.g., with a nonlinear stretching such as a classical histogram equalization) which can no longer satisfy the first and foremost preservation of spectral distance criterion.

These two contradictory criteria have been used in [23] for estimating an informative color mapping, allowing the efficient visualization of hyperspectral images. In the model proposed by the authors, these two criteria are exploited by explicitly setting the preservation of spectral distance as a 3-D mapping problem decomposed into two steps: a 2-D projection as a partial solution using classical PCA and a linear programming method to solve for the 1-D coordinate in the third dimension with the separability of features (or contrast) criterion as a set of constraints. In their framework, their method remains mainly linear. Furthermore, this two-step dimensionality-reduction method closely depends on a preprocessing step which first uses a classical PCA to a few bands (20 in their application) and an initial segmentation using a nonoptimal median-cut algorithm.

Another strategy, proposed in this paper, is to directly cast this visualization problem as a nonlinear bicriteria optimization problem including an internal parameter expressing the tradeoff or the balance between these two contradictory criteria. In this nonlinear dimension reduction framework, our bicriteria optimization problem can also be viewed as a classical Bayesian optimization strategy formalizing a tradeoff between fidelity to the unreduced (raw) spectral data (via the preservation of spectral distance-based likelihood energy term) and the expected highly contrasted solution (via the separability of features prior energy term). In this context, we will show that a hybrid optimization in several steps, combining a global and deterministic optimization procedure and a local stochastic search using the Metropolis criterion, can be exploited to efficiently minimize this complex nonlinear objective cost function related to this model.

The remainder of this paper is organized as follows. Section II describes the quantitative metrics of good visualization and the proposed dimensionality-reduction model. Section III describes the optimization strategy used to minimize the objective cost function related to our model. Finally, Section IV presents the quantitative metrics used to validate our algorithm and a set of experimental results and comparisons with existing multidimensional visualization techniques.

\section{Proposed Dimensionality-Reduction Model}

\section{A. Quantitative Metrics Of Good Visualization}

To evaluate the preservation of distances criterion of a color display algorithm, we will use in the following the correlationbased metric $\rho$ proposed in [10], [23] which is simply the correlation of the Euclidean distance between each pairwise spectral vectors in the hyperspectral image (let $X$ be this vector) and their corresponding (pairwise) Euclidean distances (color difference) in the perceptual LAB color space (let $Y$ be this vector)

$$
\rho=\frac{X^{t} Y /|X|-\bar{X} \bar{Y}}{\operatorname{std}(X) \cdot \operatorname{std}(Y)}
$$

where $X^{t},|X|, \bar{X}$, and $\operatorname{std}(X)$ denote the transpose, cardinal, mean, and standard deviation of $X$, respectively. In practice, we 
consider a subsampling of pairs of pixels in the image (i.e., all pairs with horizontal or vertical (possible) displacements of $2^{p}$ pixels for $p \leq 9$ ). In the absence of perceptual error and, thus, with no loss of information in this dimensionality-reduction problem, the ideal correlation metric is 1 . In order to also measure the separability of features or contrast criterion, we use, as in [23], the average distance between each pair of pixels in perceptual color space (it should be as large as possible): $\delta=|Y|_{1} /|Y|$ where $|Y|_{1}$ and $|Y|$ denote the $\mathrm{L} 1$ norm and the cardinal of vector $Y$, respectively.

\section{B. Bicriteria-Optimization-Approach-Based Model}

Let us define the notations used throughout this paper. We consider a hyperspectral image as a cube or a 3-D array of observed pixels made up of several 2-D arrays. We define an image by $I$ or $I(s, k)$, where $s$ indicates the spatial location and $k$ indexes the particular spectral band in which the pixel lies in the hyperspectral cube. We will use the term image slice to denote a 2-D array for a given $k$, i.e., $I(\cdot, k)$ where $N$ is the number of pixels in each image slice. We will use the term spectral vector to denote all pixels associated with a site $s$, i.e., $I(s, \cdot) \in R^{K}$, where $K$ is the number of spectral bands.

In order to provide perceptually meaningful visualizations, the main goal of our color visualization strategy is to preserve, as first and foremost criterion (and as much as possible), the distance between the spectral vector of each pair of pixels and their final perceptual color distance in the final displayed color image. As perceptual color space, we use the classical CIE 1976 $L^{*}, a^{*}, b^{*}(\mathrm{LAB})$ color space which is approximately perceptually uniform (i.e., the same amount in a color value produces a change of about the same visual importance). The second criterion is related to the contrast notion or the separability of features and is, by definition, expressed by the average distance between each pair of pixels in LAB space.

To this end, let $\mathbf{u}$ be the 3 -D vector $\left(\mathbf{u}=\left(\begin{array}{lll}L A & A\end{array}\right)^{t}\right)$ corresponding to the three $L, A, B$ color bands of the final image to be displayed. The Euclidean distance has been chosen between the spectral vector of each pair of pixels and their final perceptual color distance (first criterion) along with the average (mean) distance between each pair of pixels in LAB space (second criterion). In this setting, the visualization technique then seeks to find $\hat{\mathbf{u}}=(\hat{L} \hat{A} \hat{B})^{t}$ that minimizes the following bicriteria objective function:

$$
\begin{aligned}
\hat{\mathbf{u}}=\arg \min _{\mathbf{u}} \sum_{s, t_{s \neq t}}\left\{\left(\beta_{s, t}-\right.\right. & \left.\left\|\phi\left(\mathbf{u}_{s}\right)-\phi\left(\mathbf{u}_{t}\right)\right\|_{2}\right)^{2} \\
& \left.-\gamma\left\|\phi\left(\mathbf{u}_{s}\right)-\phi\left(\mathbf{u}_{t}\right)\right\|_{2}^{2}\right\}
\end{aligned}
$$

where the summation $\sum_{s, t_{s \neq t}}$ is over all the pairs of sites (i.e., for all sites $s$ and for all the pairs of sites including $s$ ) existing in the final image to be displayed. $\beta_{s, t}$ denotes the Euclidean distance associated between two spectral vectors, associated with the pair of sites or spatial locations $s, t$. In this model, the set of $\beta_{s, t},\left(\left\{\boldsymbol{\beta}_{s, t}\right\}\right)$ represents the observed data. The first term is related to the preservation of spectral distance criterion, and the second corresponds to the contrast or separability of features criterion. $\gamma$ is the value controlling the contribution of these two criteria. $\phi\left(\hat{\mathbf{u}}_{\mathbf{s}}=\left(\hat{L}_{s} \hat{A}_{s} \hat{B}_{s}\right)^{t}\right)$ is the function that takes into account the possible saturation effect, for each pixel, in the finally displayed RGB color space. More precisely, this function ensures the three following operations. First, it converts the $L A B$ color values into $R G B$ color values. Second, it ensures that all converted pixels are inside the RGB color space by setting negative pixel values to 0 and those that are greater than 255 to 255 . Third, it converts back the $R G B$ color values into the LAB color space. This function enables the contradiction existing between these two criteria to be understood. A high contrast (second criterion) in the perceptual $\mathrm{LAB}$ color space can be obtained at the cost of numerous saturated pixels which can no longer satisfy the accuracy of the resulting color mapping (first criterion). There are three points worth mentioning concerning the constrained nonlinear reduction model expressed by (2).

1) As already mentioned in Section I, this bicriteria optimization problem can also be viewed as a Bayesian optimization strategy formalizing a tradeoff between goodness-of-fit and some $a$ priori expected properties of the solution. More precisely, the first term of (2) measures the fidelity to the unreduced (raw) spectral data (via a so-called likelihood energy term expressing the first criterion) and the second one encodes fidelity to some a priori properties about the desired solution, namely, a highly contrasted final color image (via a prior energy term favoring the second criterion). ${ }^{1}$

2) For $\gamma=0$ and $\phi(\mathbf{u})=\mathbf{u}$ [see (2)], the function to be minimized is also the so-called stress function used as criterion in the nonlinear dimensionality-reduction model based on multidimensional scaling (MDS) [25], [26]. MDS attempts to find an embedding from the initial feature vectors in the high-dimensional space such that pairwise distances are preserved in a low dimensional space.

3) Finally, it is also worth mentioning than the objective functions to be minimized can be viewed as a Gibbs energy field related to a nonstationary Markov random field (MRF) model defined on a complete graph with longrange pairwise interactions, i.e., binary cliques $\langle s, t\rangle$ (or pairwise of pixels). Each binary clique of this MRF model is associated to a nonstationary potential since this model is spatially variant and depends on several factors such as the distance between the spectral vectors associated to each pair of pixels $s, t$ and also the value of $\phi$.

In this context, our dimensionality-reduction model is cast as a global optimization problem of a complex (nonconvex) cost function with several local extrema over the LAB color value space. In order to find a particular configuration of $\hat{\mathbf{u}}$, which efficiently minimizes this complex energy function, we use the optimization strategy in several stages described in the next section.

\footnotetext{
${ }^{1}$ Let us note that our energy-based model could not be formulated in reverse, i.e., with a contrast term as the main focus (likelihood energy term) which would be optimized under the preservation of distance criterion acting as a regularization constraint (regularization energy term) since our contrast term does not depend on the observed data $\left(\left\{\boldsymbol{\beta}_{s, t}\right\}\right)$. In our case, the contrast term is a prior or an a priori term in the Bayesian sense and is used to model some a priori properties about the solution image (more or less contrasted).
} 


\section{Optimization StRATEGY}

\section{A. First Step}

To this end, let $E_{0}$ be a simplified version of our energy function [expressed by (2)] obtained with $\gamma=0$ and $\phi(\mathbf{u})=\mathbf{u}$ (i.e., with $\phi$ being the identity function $\mathcal{I}$ ). In this context, $E_{0}$ is equivalent to the loss function also used in MDS methods to estimate a low-dimensional mapping of high-dimensional data, and two possible strategies, specifically well suited for large-scale applications (i.e., for large sample size and/or highdimensional data), exist. The first one is to use the multiresolution dimensionality-reduction model proposed in [24], and the second one is to use the so-called FastMap algorithm [27] (or its variants [28]). It was reported in [28] and [29] that the FastMap algorithm is as efficient as the best existing scalable MDS, the so-called "Landmark MDS," in terms of accuracy and speed comparisons, for very low embedding dimensions and linear Euclidean distance (which is the case in our model). For this initialization step, two MDS-based optimization strategies remain possible. The most natural strategy consists in the use of the MDS algorithm directly for estimating a three-dimensional (3-D) embedding, i.e., for the 3-D mapping $\mathbf{u}=(L A B)^{t}$ with

$$
\hat{\mathbf{u}}=\arg \min _{\mathbf{u}} \sum_{s, t_{s \neq t}}\left(\boldsymbol{\beta}_{s, t}-\left\|\mathbf{u}_{s}-\mathbf{u}_{t}\right\|_{2}\right)^{2} .
$$

The second strategy consists in the use of the MDS algorithm for the separate estimation of $L$ and $A$ and $B$ and each (1-D) mapping being defined, in our case, for the three equal-sized subsets covering the overall available wavelengths of the original hyperspectral image, i.e.,

$$
\left\{\begin{array}{l}
L=\arg \min \sum_{s, t_{s \neq t}}\left(\beta_{s, t}^{\left[1: \frac{1}{3} K\right]}-\left(L_{s}-L_{t}\right)\right)^{2} \\
A=\arg \min \sum_{s, t_{s \neq t}}\left(\beta_{s, t}^{] \frac{1}{3} K: \frac{2}{3} K\right]}-\left(A_{s}-A_{t}\right)\right)^{2} \\
B=\arg \min \sum_{s, t}\left(\beta_{s, t}^{] \frac{2}{3} K: K\right]}-\left(B_{s}-B_{t}\right)\right)^{2}
\end{array}\right.
$$

where $\beta_{s, t}^{\left[k_{0}: k_{1}\right]}$ denotes the Euclidean distance between the spectral bands $k_{0}$ and $k_{1}$ and $L_{s}, A_{s}$, and $B_{s}$ are the $L, B$, and $A$ components, respectively, at site (or pixel) $s$.

Table I summarizes the measures of good visualization of the final color mapping based on $\rho$ and $\delta$ (see Section II-A), on the simplified version of our energy function [see (3) and (4)], for the $M_{4} \mathrm{ICD}[24]$ and the FastMap [27] methods, respectively, for the 3-D mapping $\mathbf{u}=(L A B)^{t}$ (dimension of the embedding: $\operatorname{dim}=3)$ and for the separate estimation of $L$ and $A$ and $B(\operatorname{dim}=3 \times 1)$. Experiments show that the FastMap [27] is less accurate than the $M_{4} \mathrm{ICD}[24]$ and that the separate estimation of $L$ and $A$ and $B$ leads to the best results for the FastMap method. In accordance with our opinion, this may be due to the fact that the FastMap is all the more efficient that the embedding is achieved at very low dimensions [28]. This greatly simplifies the FastMap procedures and, thus, its reliability. Although somewhat less accurate than the dimensionalityreduction model presented in [24], the FastMap method has the advantage of being about ten times faster compared with the
TABLE I

COMPARISON OF CORRELATION $\rho$ AND AVERAGE DisTANCE $\delta$ FOR THE FastMap AND THE MultiResolution $M_{4}$ ICD MOdel For THE SiMPLIFIED VERSION OF OUR ENERGY FUNCTION

\begin{tabular}{||c||c|c|c||}
\hline \hline$\rho_{[\delta]}$ & FastMap dim=3 & FastMap dim=3×1 & $\mathrm{M}_{4} \mathrm{ICD}_{1}$ \\
\hline \hline Moffett $_{01}$ & $0.827_{[12.9]}$ & $0.931_{[10.0]}$ & $0.975_{[10.3]}$ \\
\hline Moffett $_{02}$ & $0.922_{[9.12]}$ & $0.960_{[10.5]}$ & $0.981_{[11.1]}$ \\
\hline Lun.lake $_{01}$ & $0.942_{[8.13]}$ & $0.998_{[11.1]}$ & $0.983_{[12.4]}$ \\
\hline Lun.lake $_{02}$ & $0.863_{[11.9]}$ & $0.976_{[13.0]}$ & $0.960_{[14.2]}$ \\
\hline Cuprite $_{01}$ & $0.947_{[9.10]}$ & $0.988_{[11.4]}$ & $0.975_{[12.5]}$ \\
\hline Cuprite $_{02}$ & $0.913_{[11.4]}$ & $0.986_{[13.9]}$ & $0.978_{[18.3]}$ \\
\hline Jasper $_{01}$ & $0.893_{[11.1]}$ & $0.918_{[9.8]}$ & $0.965_{[11.6]}$ \\
\hline Jasper $_{02}$ & $0.988_{[4.24]}$ & $0.942_{[7.5]}$ & $0.961_{[7.3]}$ \\
\hline Jasper $_{03}$ & $0.851_{[12.9]}$ & $0.945_{[9.0]}$ & $0.980_{[11]}$ \\
\hline Jasper $_{04}$ & $0.804_{[11.1]}$ & $0.954_{[8.2]}$ & $0.975_{[7.9]}$ \\
\hline Jasper & $0.996_{[4.70]}$ & $0.952_{[7.2]}$ & $0.973_{[6.9]}$ \\
\hline \hline Average & $\mathbf{0 . 9 0 4}_{[9.7]}$ & $\mathbf{0 . 9 5 8}_{[\mathbf{1 0 . 1}]}$ & $\mathbf{0 . 9 7 4}_{[11.2]}$ \\
\hline \hline
\end{tabular}

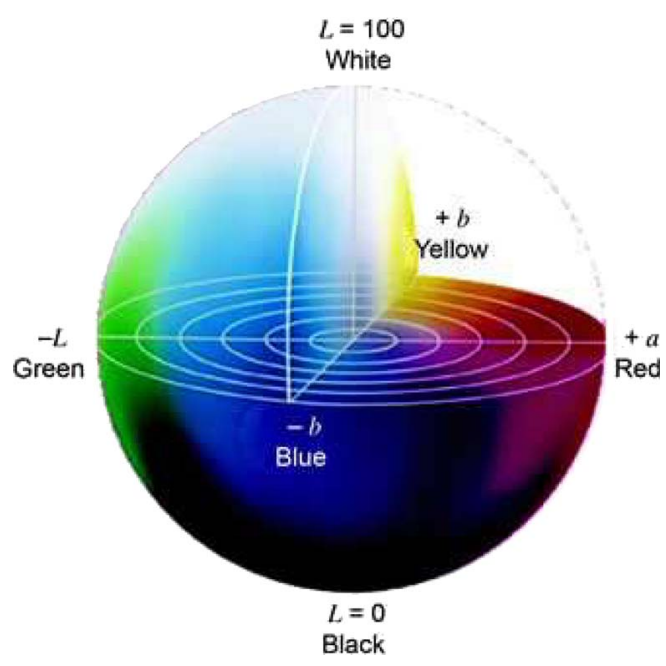

Fig. 1. CIE Lab color space. $L$ is always positive and represents brightness. $a>0$ represents the red component, $a<0$ represents the green component, $b>0$ represents the yellow component, and $b<0$ represents the blue component.

multiresolution method described in [24]. For this reason and also due to the fact that, at this stage, we only need a good initialization (related to a simplified version of our bicriteria energy function which will be refined later to find a solution related to our complete energy-based model), we decided to use the FastMap algorithm (for the separate estimation of $L$ and $A$ and $B$ mappings) as an interesting compromise between accuracy and speed.

It is important to mention that, at this stage, we are not assured that the LAB color values of the 3-D mapping $\mathbf{u}$ are not saturated in the RGB space. In order to fix this problem, we use a simple linear stretching of the $L, A$, and $B$ color values such as $L \in[0: 100]$ and such that $A, B$ have a maximal amplitude of 100 with a zero mean in order to ensure that a very small minority of pixels are outside the RGB color space (see Fig. 1). Mathematically, this helps ensure that $\phi\left(\mathbf{u}_{s}\right)=\mathbf{u}_{s}$ for all sites $s$ and, concretely, that there are no saturated pixels which could alter the preservation of spectral distance criterion. 
At this stage, this linear stretching allows us to find the color mapping that minimizes the following objective function:

$$
\hat{\mathbf{u}}=\arg \min _{\mathbf{u}} \sum_{s, t_{s \neq t}}\left(\boldsymbol{\beta}_{s, t}^{\text {scaled }}-\left\|\phi\left(\mathbf{u}_{s}\right)-\phi\left(\mathbf{u}_{t}\right)\right\|_{2}\right)^{2}
$$

where $\phi\left(\mathbf{u}_{s}\right) \approx \mathbf{u}_{s}$ due to our suitable range of LAB color values and $\boldsymbol{\beta}_{s, t}^{\text {scaled }}=\rho \boldsymbol{\beta}_{s, t}$ with $\rho$ as a scaling factor ensuring that, after the linear stretching, the pairwise distances of the 3-D mapping $\mathbf{u}$ and the distance of the pairwise spectral vectors are still preserved. This scaling factor is defined by

$$
\rho=\frac{\sum_{s, t} d_{s, t}^{\text {stretch }}}{\sum_{s, t} d_{s, t}}
$$

where $d_{s, t}$ and $d_{s, t}^{\text {stretch }}$ are the sets of pairwise Euclidean distances of the image before and after the linear stretching process, respectively. In order to decrease the computational load, in our application, the summation integrates to a subsampled (with a factor of ten pixels in length and in width) set of pixel pairs in the two images.

\section{B. Second Step}

At this stage, an increase in the contrast of the image (via the minimization of the second energy term) related to our second criterion of our bicriteria optimization problem may be obtained by multiplying the LAB color values by a spatially variant positive scaling factor (and at a price that some pixels will become saturated in the RGB space and will no longer satisfy the preservation of spectral distance criterion). It is worth mentioning that this scaling factor is necessarily spatially varying because it is closely related to the $\mathrm{LAB}$ color values which spatially vary in the image. However, at this stage, we can approach the solution and give a rough estimation to our optimization problem by estimating the spatially invariant scaling factor $k$ for which

$$
\begin{array}{r}
\hat{k}=\arg \min _{k} \sum_{s, t_{s \neq t}}\left\{\left(k \boldsymbol{\beta}_{s, t}^{\text {scaled }}-\left\|\phi\left(k \mathbf{u}_{s}\right)-\phi\left(k \mathbf{u}_{t}\right)\right\|_{2}\right)^{2}\right. \\
\left.-\gamma\left\|\phi\left(k \mathbf{u}_{s}\right)-\phi\left(k \mathbf{u}_{t}\right)\right\|_{2}^{2}\right\} .
\end{array}
$$

In order to do this estimation, we use a simple local discrete grid search routine, for the parameter $k$ in a suitable range $(k \in$ $[1.0-3.0]$ with a fixed step size set to 0.2$)$. In order to decrease the computation time, we do not consider a complete graph but each node (or pixel) is connected with its four nearest neighbors and four equally spaced other pixels located within a square neighborhood window of fixed size $N_{s}=61$ pixels centered around the pixel (for an input image assumed to be toroidal, i.e., wrapping around at the edges).

Since the aforementioned process can saturate some pixels in the RGB space (particularly for large values of $\gamma$ ) and that may alter the optimal value of $k \boldsymbol{\beta}_{s, t}^{\text {scaled }}$, we refine the estimation of this parameter with a least square estimation. More precisely, $k \boldsymbol{\beta}_{s, t}^{\text {scaled }}$ is corrected by the factor [28]

$$
\rho=\frac{\sum_{s, t} \hat{\beta}_{s, t}^{2} / \beta_{s, t}^{2}}{\sum_{s, t} \hat{\beta}_{s, t} / \beta_{s, t}}
$$

where $\beta_{s, t}$ is the true (unembedded) distance, using the set of pairwise spectral vectors of the hyperspectral image, and $\hat{\beta}_{s, t}$ is the estimated (in the embedded space) distance, using the set of pixel pairs in the stretched $\mathbf{u}$ mapping. In order to decrease the computation time, we use a subsampling of ten pixels in length and width.

\section{Third Step}

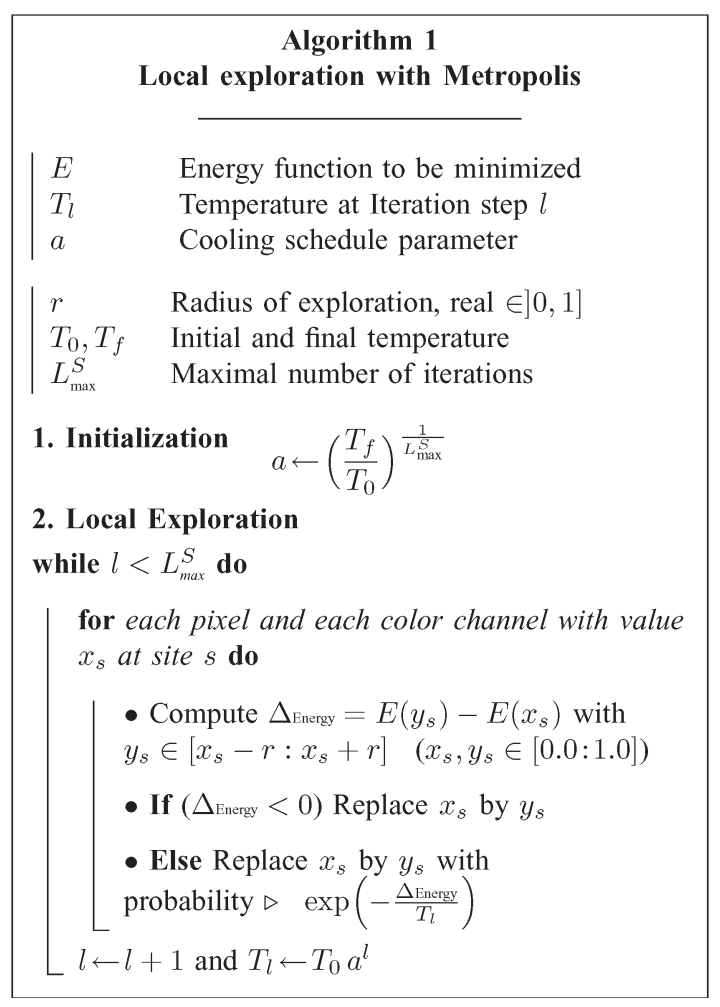

At this stage, we are very close to the solution of our optimization problem, and this optimization problem could be reduced to only optimize the value of the set of saturated pixels in order to satisfy, as much as possible, the preservation of spectral distance criterion. We decided to refine the estimation given by the aforementioned deterministic optimization method, and to this end, we use the previous optimization result as the initialization of a stochastic local search for our complete energy function. Practically speaking, we use a local exploration around the current solution using the Metropolis criterion [30] and a low radius of exploration (see Algorithm 1). In order to decrease the computational load, we consider, for this step, the same simplified graph, mentioned in Section III-B, in which each node is connected with its four nearest neighbors and four $\left(N_{c}=4\right)$ other sites equally spaced and located within a square neighborhood window of size $N_{s}=61$ centered around the pixel. Fig. 2 shows the spatial neighborhood used in our model.

Two internal parameters are sensitive and crucial for this last procedure, namely, the radius of exploration $r$ and, in a least measure, the starting temperature $T_{0}$ of the local stochastic search. The first parameter was set in order to locally explore a solution whose color values are close to the initial solution given by the preceding deterministic minimization procedure (a value $r=0.04$ ensures that the final solution will exhibit 


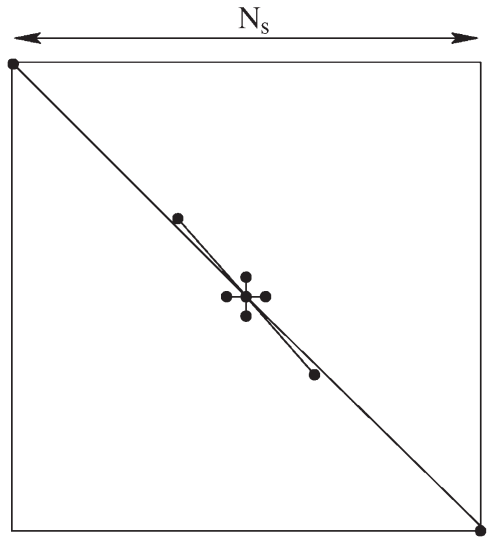

Fig. 2. Spatial neighborhood used in our model. Each pixel is connected with its four nearest neighbors and $N_{c}=4$ equally spaced other pixels located within a square neighborhood window of fixed size $N_{s}=61$ pixels, i.e., the first pixel of this square, the 1240th $\left(\left(N_{s}^{2} / 3\right)\right.$ th) pixel (20th row and 20th column), the 2480th $\left(\left(2 N_{s}^{2} / 3\right)\right.$ th pixel (41th row and 40th column), and the last pixel or the $(61 \times 61)$ th pixel.

output channel values, centered around the gradient estimation $\pm 0.04 * 100= \pm 4$ channel values for a final LAB image whose channel values are comprised in $[0: 100]) . T_{0}$ is set in order to ensure that, at the beginning of the stochastic search, approximately one-third of sites change their luminance values between two complete image sweeps. $T_{f}$, which is the final temperature, can be easily found in our case, since a good final temperature for a simulated annealing-like minimization procedure has to ensure that, at the end of the stochastic search, very few sites change their channel color values between two complete image sweeps. In our algorithm, this parameter has been easily found after a few trials. We have found that $T_{0}=$ 1 and $T_{f}=10^{-3}$ were appropriate for all the experiments presented in this paper. ${ }^{2}$ Due to the small radius of exploration, the computational complexity of this optimization step (in fact, a simple local search around the scaled MDS estimation) is considerably reduced, and this explains why a low number of iterations $L_{\max }^{S}=50$ is herein performed. Fig. 3 shows us the evolution of the energy function for this stochastic local search using the Metropolis criterion.

\section{EXPERIMENTAL RESUlts}

\section{A. Setup and Hyperspectral Image Used}

In all the experiments, we have thus considered the following setup. For the preprocessing model, we exclude the spectral bands containing mainly noise, and to this end, we use the preprocessing strategy described in [24].

\footnotetext{
${ }^{2}$ Let us note that if $T_{0}=T_{f}=0$, this optimization step becomes a simple deterministic optimization procedure such as a classical gradient descent with a sampling strategy. Let us also add that, in practice, it is easy to find a procedure that automatically (and adaptively for each hyperspectral image) estimates $T_{0}$, ensuring a $30 \%$ ratio of site changes between two complete image sweeps. It simply consists in starting from an upper bound for this initial temperature, namely, $T_{0}=10$, and in iteratively decreasing this initial temperature (e.g., by $10 \%$ ) and performing one iteration of the local stochastic search, until the ratio of site changes (between two complete image sweeps) is below 30\%. Given this initial estimate for $\hat{T}_{0}$, we can then start the initialization step of our local exploration search along with our classical geometric temperature cooling schedule (cf. Algorithm 1).
}

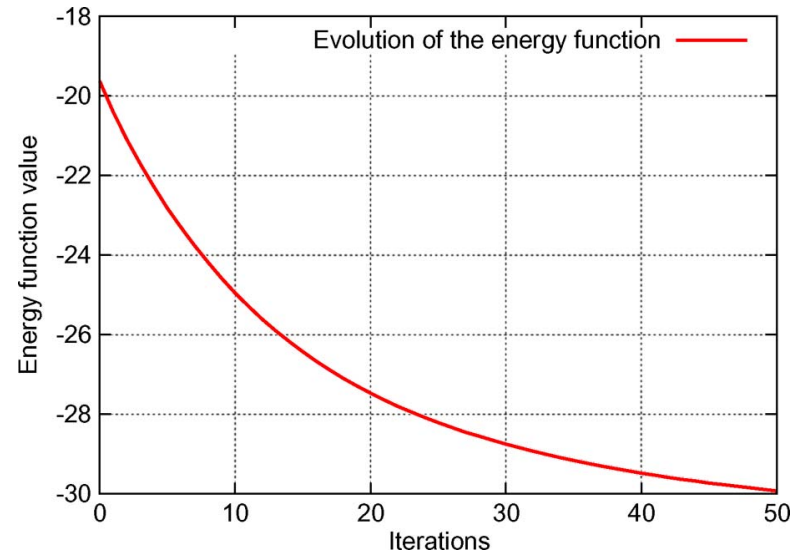

Fig. 3. Evolution of the energy function for the third step (stochastic local search using the Metropolis criterion) of our multistage optimization scheme (AVIRIS image LUNARLAKE1.RFL with $\gamma=0.1$ ).

The hyperspectral cubes chosen for our experimental results are from the National Aeronautics and Space Administration Jet Propulsion Laboratory Airborne Visible/Infrared Imaging Spectrometer (AVIRIS) system [32], which captures 224 spectral bands, ranging from 400 to $2500 \mathrm{~nm}$ with a 1995 AVIRIS. The image size is usually around $512 \times 614$ pixels. We have used the reflectance data which are atmospherically corrected to compensate for absorption and the spectrum of the sun. The AVIRIS data are generously available for download online at HTTP address http://aviris.jpl.nasa.gov/html/aviris.free data.html.

\section{B. Quantitative Comparison and Discussion}

First, we have tested and evaluated our algorithm (called BCOCDM for bicriteria optimization for color display model) with $\gamma=0$, i.e., with the best possible accuracy (i.e., without the second energy term related to the contrast criterion which possibly alters the first and foremost criterion), and we have computed our two metrics of good visualization for different existing color display techniques. These techniques are the $M_{4} \mathrm{ICD}$ [24], the one proposed in [23], the color matching function (CMF) [10], the classical PCA method with a final linear scaling, the PCA with outlier reduction $\left(\mathrm{PCA}_{2} \%\right.$ ) (which scales the final bands with $2 \%$ of the pixels at the ends of each channel being saturated in order to enhance the contrast), and finally, the PCA with histogram equalization $\left(\mathrm{PCA}_{h}\right)$. Results are shown in Table II. Display results with comparisons with other techniques are shown in Figs. 4 and 5.

The comparison of results shows that $\mathrm{PCA}_{2} \%$ and $\mathrm{PCA}_{\mathrm{h}}$ have a good score for the separability of features $(\delta)$, i.e., a good contrast in the final displayed image. Nevertheless, this color contrast enhancement is made to the detriment of the overall correlation score which indicates that the color values of each channel of the final color image are strongly biased and the resulting image (i.e., its color values) is far less informative than those of the other visualization techniques. Our method gives a very well performing average score of preservation of distance between all the existing visualization techniques with a contrast value comparable with that of the classical PCA 
TABLE II

COMPARISON OF CORRELATION $\rho$ AND CONTRAST $\delta$ FOR OUR BCOCDM ALGORITHM (WiTH $\gamma=0$ ) AND THE OTHER EXISTING COLOR DisPlay MODELS

\begin{tabular}{|c|c|c|c|c|c|c|c|}
\hline$\rho_{[\delta]}$ & BCOCDM & $\mathrm{M}_{4} \mathrm{ICD}[24]$ & [23] & CMF (in [23]) & PCA (in [23]) & $\mathrm{PCA}_{2 \%}$ (in [23]) & $\mathrm{PCA}_{\mathrm{h}}$ (in [23]) \\
\hline Moffett $_{01}$ & $\mathbf{0 . 9 1 8}_{[14.4]}$ & $0.975_{[10.3]}$ & $0.96_{[38]}$ & $0.94_{[16]}$ & $0.91_{[13]}$ & $0.68_{[50]}$ & $0.46_{[81]}$ \\
\hline Moffett $_{02}$ & $\mathbf{0 . 9 3 5}_{[15.9]}$ & $0.981_{[11.1]}$ & $0.96_{[54]}$ & $0.79_{[31]}$ & $0.92_{[12]}$ & $0.51_{[48]}$ & $0.35_{[77]}$ \\
\hline Moffett $_{03}$ & $\mathbf{0 . 9 4 8}[13.2]$ & $0.981_{[8.1]}$ & $0.93_{[25]}$ & $0.69_{[30]}$ & $0.96_{[10]}$ & $0.68_{[45]}$ & $0.41_{[76]}$ \\
\hline Lun.lake $_{01}$ & $\mathbf{0 . 9 8 2 _ { [ 1 3 . 1 ] }}$ & $0.983_{[12.4]}$ & $0.95_{[51]}$ & $0.82_{[6]}$ & $0.92_{[11]}$ & $0.53_{[52]}$ & $0.21_{[85]}$ \\
\hline Lun.lake $_{02}$ & $\mathbf{0 . 9 7 5}[15.4]$ & $0.960_{[14.2]}$ & $0.84_{[60]}$ & $0.81_{[7]}$ & $0.95_{[16]}$ & $0.37_{[44]}$ & $0.27_{[80]}$ \\
\hline Cuprite $_{01}$ & $\mathbf{0 . 9 7 9}_{[13.2]}$ & $0.975_{[12.5]}$ & $0.90_{[52]}$ & $0.87_{[5]}$ & $0.91_{[11]}$ & $0.55_{[48]}$ & $0.32_{[81]}$ \\
\hline Cuprite $_{02}$ & $\mathbf{0 . 9 7 5}[16.8]$ & $0.978_{[18.3]}$ & $0.91_{[73]}$ & $0.88_{[8]}$ & $0.95_{[14]}$ & $0.43_{[53]}$ & $0.28_{[81]}$ \\
\hline Average & $0.958_{[14.6]}$ & $0.976_{[12.4]}$ & $0.92_{[50.4]}$ & $0.83_{[14.7]}$ & $0.93_{[12.4]}$ & $0.54_{[48.5]}$ & $0.33_{[80.1]}$ \\
\hline
\end{tabular}
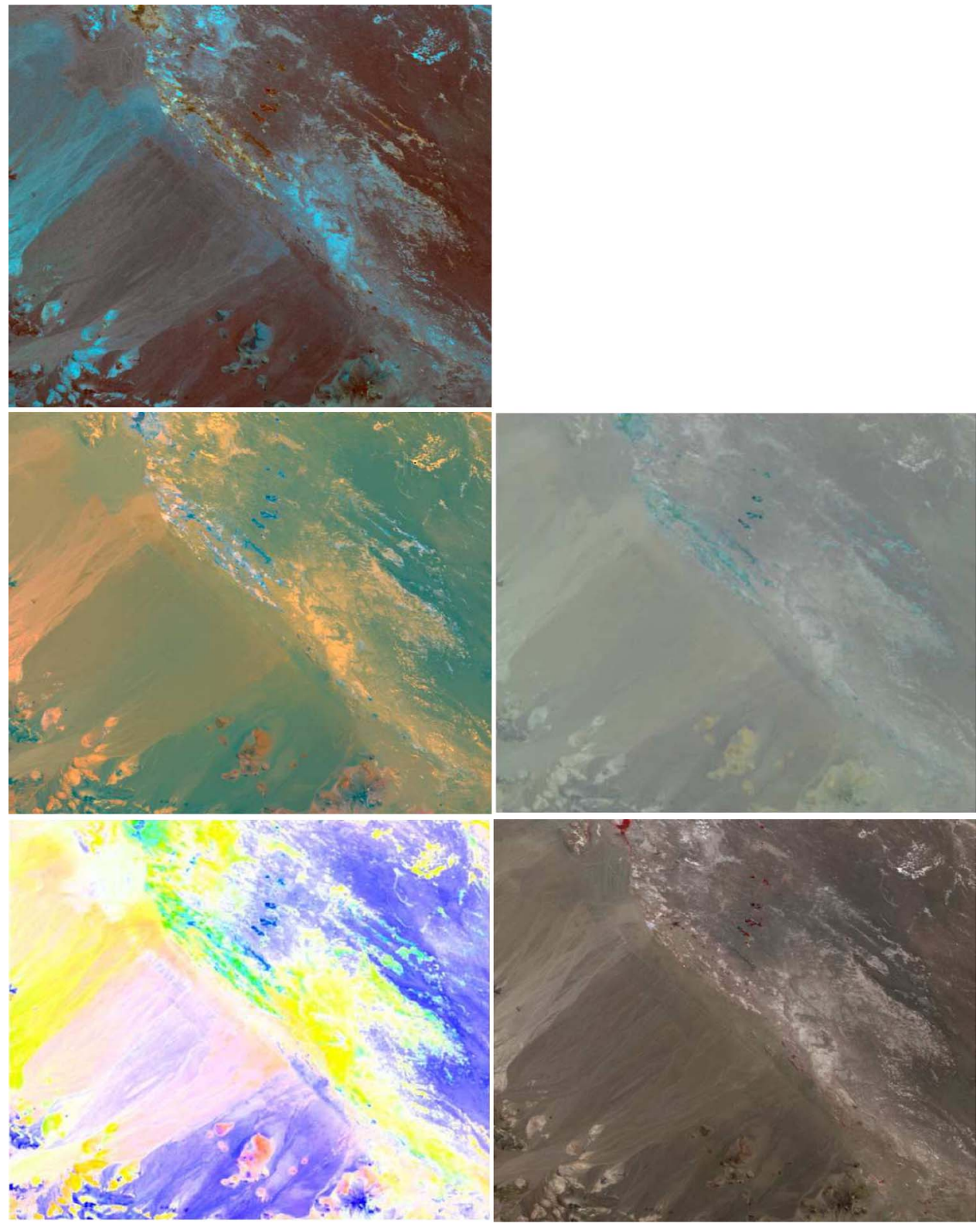

Fig. 4. Comparison of color display results on the AVIRIS image LUNARLAKE 01 obtained (in lexicographical order) by our BCOCMD model [0.98 $8_{[13.1]}(\gamma=$ 0.0)] and four existing state-of-the-art visualization models, namely, the M4ICD [24] $\left(0.98_{[12.4]}\right)$, the CMF [10] $\left(0.82_{[6]}\right)$, the one proposed in [23] (0.95 $\left.{ }_{[51]}\right)$, and a false-color composite (CIR) image [31] in which the near-infrared band (spectral band number 53;0.858 $\mathrm{mm}$ ) is displayed in red, the red band (spectral band number $29 ; 0.646 \mathrm{~mm}$ ) is displayed in green, and the green band (spectral band number $19 ; 0.547 \mathrm{~mm}$ ) is displayed in blue $\left(0.92_{[6.9]}\right)$. 


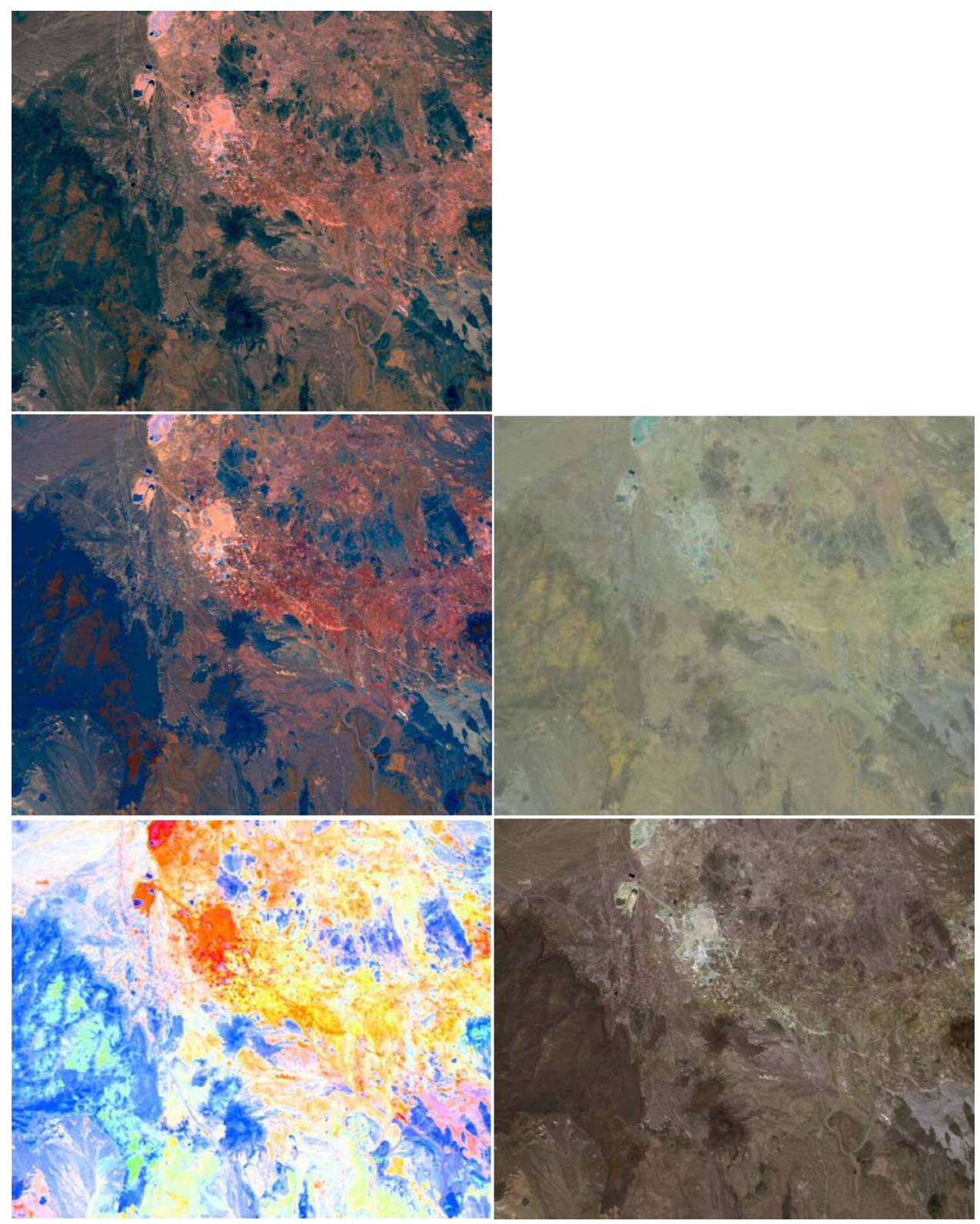

Fig. 5. Comparison of color display results on the AVIRIS images CUPRITE 02 obtained (in lexicographical order) by our BCOCMD model $\left[0.98_{[16.8]}(\gamma=\right.$ $0.0)$ ] and four existing state-of-the-art visualization models, namely, the M4ICD [24] $\left(0.98_{[18.3]}\right)$, the CMF [10] ( $\left.0.88_{[8]}\right)$, the one proposed in [23] (0.91 [73] $)$, and a false-color composite (CIR) image [31] in which the near-infrared band (spectral band number 53;0.858 mm) is displayed in red, the red band (spectral band number $29 ; 0.646 \mathrm{~mm}$ ) is displayed in green, and the green band (spectral band number $19 ; 0.547 \mathrm{~mm})$ is displayed in blue $(0.85[8.8]$ ).

technique. Another important characteristic of our method is that it provides a stable value of preservation of distance and contrast scores for a wide variety of images. Each image given by our method exploits the overall dynamic range of the color values with a good natural-looking color association and no colors "pop out" strongly. The different regions can be easily identified, and very fine details are visible without artifacts such as noise or blurring artifacts due to the reduction/compression model or spectral ringing artifacts, glints, and anomalous fluorescence hot spots (see also Fig. 6). It is worth mentioning that our algorithm allows us to automatically find the optimal $\gamma$, ensuring the best contrast metric without sensibly decreasing the maximum "preservation of distance" score. This optimal $\gamma$ can be automatically found in our case since our bicriteria visualization model can give, for each final displayed image with an increasing contrast (i.e., for each increasing discretized value of $\gamma$ starting from $\gamma=0$ ), the value of the two metrics of good visualization (as it was done for Table III or Fig. 7) and stop when the preservation of distance score starts to decrease sensibly (see Fig. 6 and the preservation of distance scores indicated in bold in Table III and corresponding to the maximum value of the contrast $\delta$ for which the distance score $\rho$ is not below $0.5 \%$ of the maximum distance score). Second, we have tested and evaluated our algorithm with increasing values of $\gamma$, thus favoring more and more the contrast enhancement or separability of features to the detriment of the overall correlation score. Results are shown in Table III. This contrast enhancement is achieved, of course, to the detriment of the accuracy 

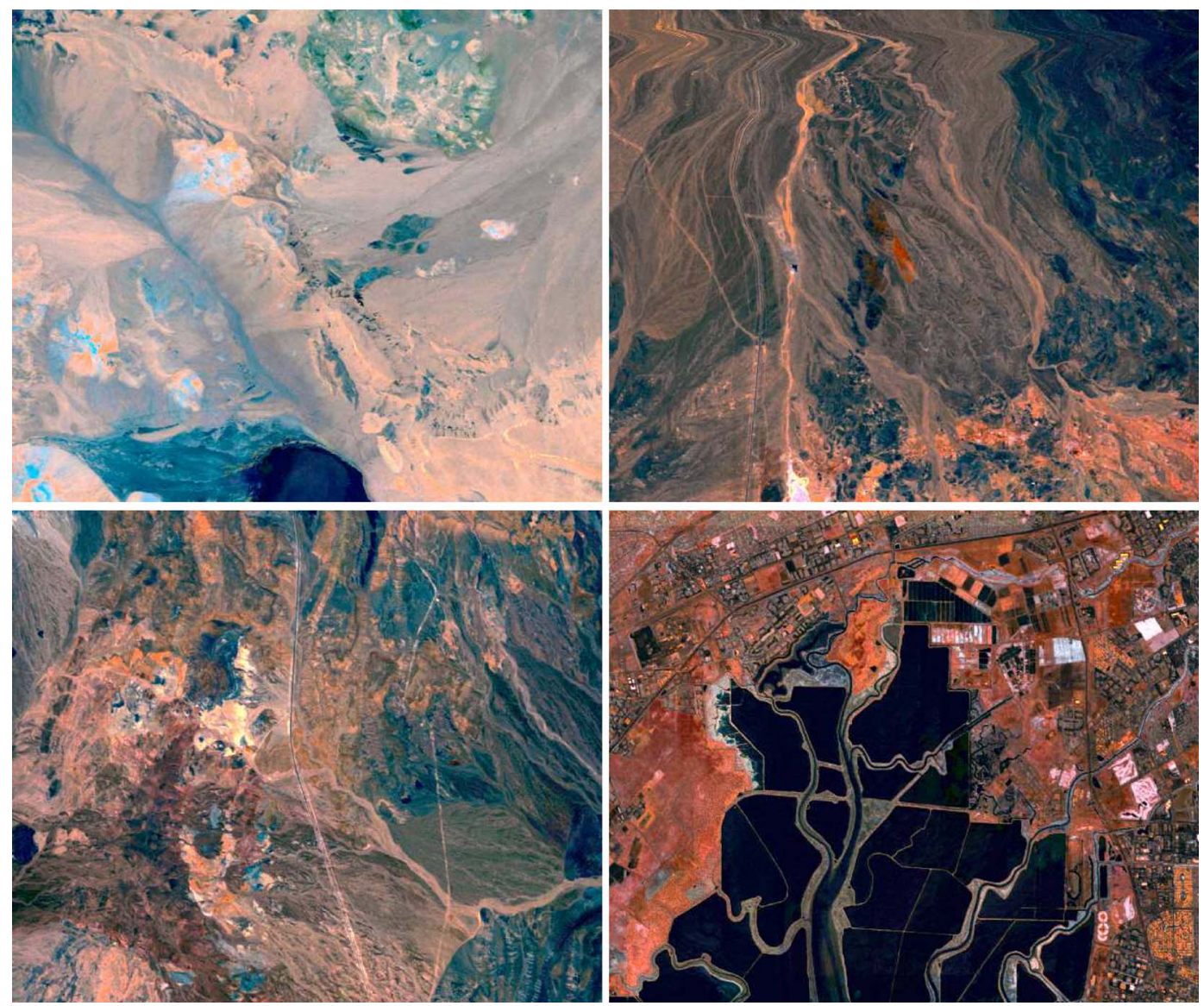

Fig. 6. Different color display results obtained by our BCOCDM algorithm with $\gamma$ being the highest value ensuring the best contrast metric without sensibly decreasing the maximum "preservation of distance" score (indicated in bold in Table III). From top to bottom and left to right, respectively, are the AVIRIS scenes LunARLAKE $02(\gamma=0.0)$, Cuprite $01(\gamma=0.1)$, Cuprite $03(\gamma=0.1)$, and MOFFETT $_{02}(\gamma=0.2)$. More color display results are publicly available at the following HTTP address: www.iro.umontreal.ca/ mignotte/ResearchMaterial/bcocdm.html.

criterion $^{3}$ but not necessarily to the computational time, since our procedure allows us, at the end of the gradient procedure, to successively increase $\gamma$ and save the resulting color visualization (see Fig. 8). This generation of the set of color displays with increasing contrast enhancement allows us to gradually distinguish the different existing materials in this scene, particularly between those that have very similar spectral signatures, thus making easier and more reliable the interpretation and quick overview of such multidimensional hyperspectral images. Indeed, we can see, in Fig. 8, that the different regions can be easily identified and very fine details are visible; for example, we can easily distinguish the small and rectangular shapes of the buildings with their concrete or shiny metal roofing (each building appears as a square of less than four or six pixels in width in these two images) with a very good edge preservation and resolution. Man-made structures and objects are clearly identifiable in these two images. Nevertheless, it is

${ }^{3}$ We recall (cf. Section II-B) that, due to the fact that the RGB color space is a subset of the perceptual CIELab color space, a high contrast (second criterion) in this perceptual color space can only be obtained at the cost of numerous saturated pixels (i.e., by setting negative RGB pixel values to 0 and those greater than 255 to 255) which may no longer satisfy the first accuracy criterion. Consequently, this correction (creating some saturated pixels) is necessarily done as soon as the best possible "preservation of distance" score begins to decrease (and that the second criterion is favored via a high value of the regularization parameter $\gamma$ ). Moreover, this correction is done all the more often (quantitatively) than the "distance" score is far from its best value. worth recalling that the resulting high contrast enhancement shown on the three right images of Fig. 8 is achieved to the detriment of the preservation of distance or correlation score (first criterion of accuracy). For example, for the top-middle image (namely, Moffet 01 with $\gamma=0.4$ ), the contrast enhancement is achieved, relatively to the top-left image $(\gamma=0.2)$, to the detriment of $2.2 \%$ (i.e., $0.893-0.871=0.022$, see Table III) of pairs of pixels which can no longer satisfy the agreement between the distance of spectrums (associated to each pair of pixels) and their perceptual color distance in the final fused image to be displayed. In other words (and relatively to the topleft image), there are $2.2 \%$ more of pairs of pixels for which the color difference (distance) is no more faithful to the distance computed from the unreduced (raw) spectral data (or 2.2\% loss of accuracy, for a contrast improvement of $63 \%$ for this topmiddle image).

It is also worth noting that if the contrast metric is very important for a specific application (e.g., target separation and/or localization, etc.), our model will also allow us to easily find the optimal regularization parameter $\gamma$ ensuring the best contrast metric for a minimum a priori fixed value for the preservation of distance score; it simply consists in running the algorithm with increasing values of $\gamma$ (as was done for the Table III or Fig. 7) and in accepting, as output mapping, the solution image related to both the maximum contrast score and that whose preservation of distance score is maintained above a minimum 
TABLE III

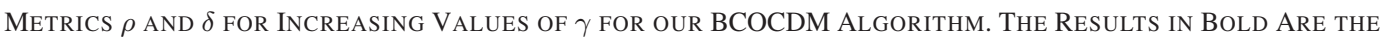
$(\rho, \delta)$ Parameter Vectors For Which the Contrast $\delta$ Is the MaXimum When the Corresponding Distance Score $\rho$ IS NOt Below 0.5\% of The MAXimum Distance Score

\begin{tabular}{|c|c|c|c|c|c|c|}
\hline$\gamma$ & 0.0 & 0.05 & 0.1 & 0.15 & 0.2 & 0.4 \\
\hline Lun.lake $_{01}$ & $0.982_{[13.1]}$ & $0.984_{[17.6]}$ & $0.971_{[20.4]}$ & $0.965_{[21.2]}$ & $0.950_{[23.0]}$ & $0.911_{[25.9]}$ \\
\hline Lun.lake $_{02}$ & $0.975_{[15.4]}$ & $0.970_{[15.9]}$ & $0.963_{[16.5]}$ & $0.955_{[17.1]}$ & $0.945_{[17.7]}$ & $0.886_{[21.1]}$ \\
\hline Cuprite $_{01}$ & $0.979_{[13.2]}$ & $0.979_{[13.8]}$ & $0.975_{[16.7]}$ & $0.960_{[19.1]}$ & $0.953_{[20.5]}$ & $0.885_{[26.0]}$ \\
\hline Cuprite $_{02}$ & $0.975_{[16.8]}$ & $0.971_{[17.5]}$ & $0.958_{[20.8]}$ & $0.941_{[24.0]}$ & $0.932_{[24.9]}$ & $0.855_{[30.0]}$ \\
\hline Cuprite $_{03}$ & $0.971_{[16.4]}$ & $0.970_{[17.0]}$ & $0.966_{[20.8]}$ & $0.948_{[24.6]}$ & $0.937_{[25.6]}$ & $0.850_{[31.3]}$ \\
\hline Cuprite $_{04}$ & $0.956_{[11.3]}$ & $0.951_{[11.8]}$ & $0.951_{[13.0]}$ & $0.945_{[16.0]}$ & $0.935_{[16.7]}$ & $0.868_{[25.7]}$ \\
\hline Moffet $_{01}$ & $0.918_{[14.4]}$ & $0.910_{[14.9]}$ & $0.902_{[15.4]}$ & $0.893_{[16.0]}$ & $0.893_{[17.4]}$ & $0.871_{[28.4]}$ \\
\hline Moffet $_{02}$ & $0.935_{[15.9]}$ & $0.930_{[16.5]}$ & $0.935_{[18.8]}$ & $0.948_{[26.5]}$ & $0.939_{[27.2]}$ & $0.895_{[38.5]}$ \\
\hline Moffet $_{03}$ & $0.948_{[13.2]}$ & $0.951_{[14.4]}$ & $0.959_{[18.8]}$ & $0.953_{[22.8]}$ & $0.946_{[23.4]}$ & $0.908_{[32.4]}$ \\
\hline Jasper $_{01}$ & $\mathbf{0 . 8 8 0}_{[14.0]}$ & $0.873_{[14.5]}$ & $0.866_{[15.1]}$ & $0.856_{[15.7]}$ & $0.844_{[16.3]}$ & $0.802_{[21.0]}$ \\
\hline Jasper $_{02}$ & $\mathbf{0 . 9 3 5}_{[10.4]}$ & $0.929_{[10.8]}$ & $0.921_{[11.3]}$ & $0.912_{[11.7]}$ & $0.901_{[12.2]}$ & $0.834_{[14.3]}$ \\
\hline Jasper $_{03}$ & $0.904_{[13.9]}$ & $\mathbf{0 . 9 0 0}[14.3]$ & $0.893_{[14.9]}$ & $0.887_{[15.4]}$ & $0.887_{[18.2]}$ & $0.844_{[36.3]}$ \\
\hline Jasper $_{04}$ & $0.907_{[12.3]}$ & $0.902_{[12.8]}$ & $0.896_{[13.3]}$ & $0.897_{[14.5]}$ & $0.897_{[16.7]}$ & $0.866_{[25.4]}$ \\
\hline Jasper $_{05}$ & $0.959_{[11.0]}$ & $\mathbf{0 . 9 5 6}[11.4]$ & $0.953_{[11.9]}$ & $0.947_{[12.3]}$ & $0.957_{[20.7]}$ & $0.900_{[32.7]}$ \\
\hline Average & $0.945_{[13.7]}$ & $0.941_{[14.5]}$ & $0.936_{[16.2]}$ & $0.929_{[18.4]}$ & $0.923_{[20.0]}$ & $0.870_{[27.8]}$ \\
\hline
\end{tabular}
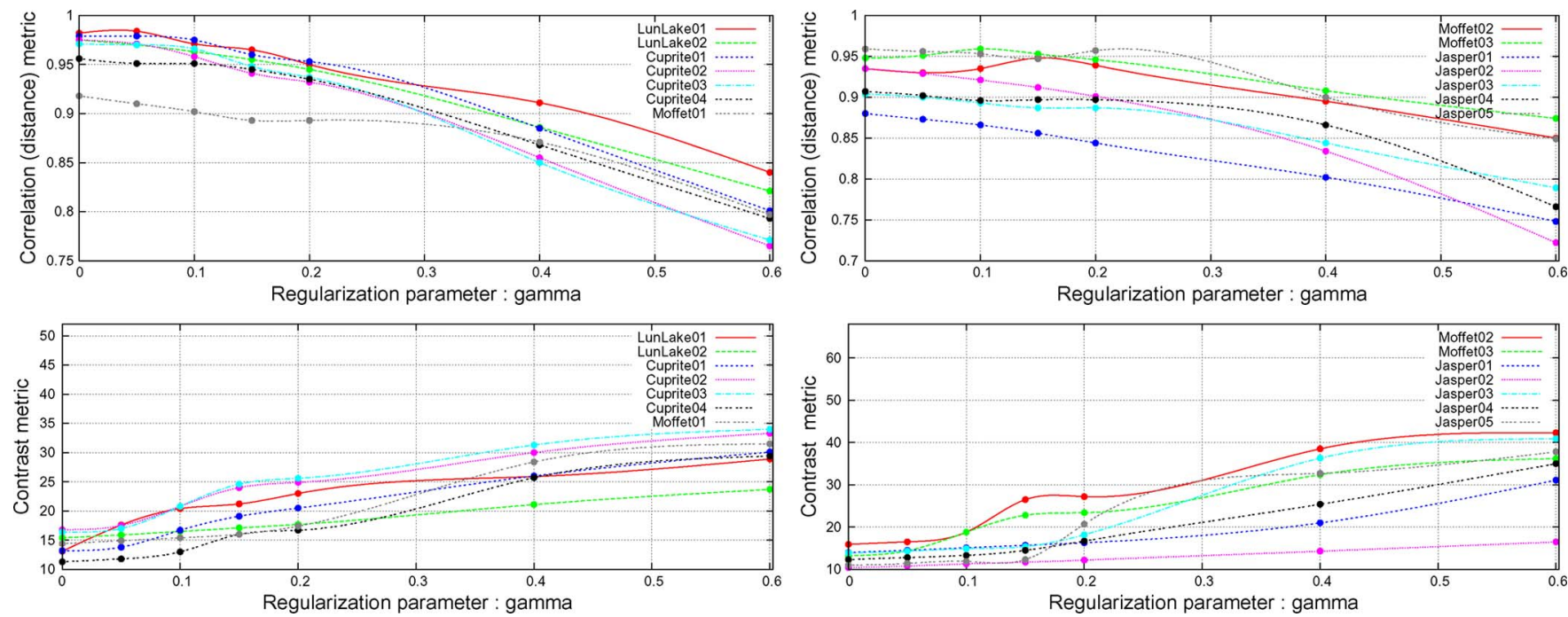

Fig. 7. Correlation metric $\rho$ (preservation of distance criteria) and contrast metric $\delta$ for increasing values of the regularization parameter $\gamma$ for our BCOCDM algorithm (see also Table III).

value. This strategy can then be used if the contrast metric is the main focus (while the distance or accuracy term has to be kept above a given minimum score).

Third, our method also has the appealing ability to slightly improve the preservation of distance score by simply increasing the number of connections of our graph (and, thus, also the computational cost of our algorithm). Presently, we recall that each node (or pixel) is connected with its four nearest neighbors and $N_{c}=4$ equally spaced other pixels located within a square neighborhood window of fixed size $N_{s}=61$ pixels centered around the pixel. Table IV shows how this score is slightly improved by increasing this number of connections on the AVIRIS images MOFFET 01 .

We have also tested the influence of variations of the size $N_{s}$ of the neighborhood window on the "preservation of distance" and contrast scores for increasing values of $\gamma$. Table $\mathrm{V}$ shows the obtained results for the LUNLAKE 01 AVIRIS scene and for some values of $N_{s}$ varying around the value given in our application (i.e., $N_{s}=61$ ). Experiments show that our model is not too sensitive to this parameter if $N_{s}$ is large enough, for example, the value of $N_{s}$ given in our application. It also appears that values of $N_{s}$ greater than 61 do not greatly improve the final results.

\section{Algorithm}

The color mapping procedure takes, on average, $1 \mathrm{~min}$ (for a $512 \times 614 \times 224$ hyperspectral AVIRIS image) for an Intel i7 CPU with $3.33 \mathrm{GHz}, 6675.25$ bogomips, and nonoptimized code running on Linux. Running times in seconds for our methods and for different images are summarized in Table VI. It must be noted than our Metropolis optimization scheme can be efficiently implemented by using the parallel abilities of a graphic processor unit (GPU) (embedded on most 

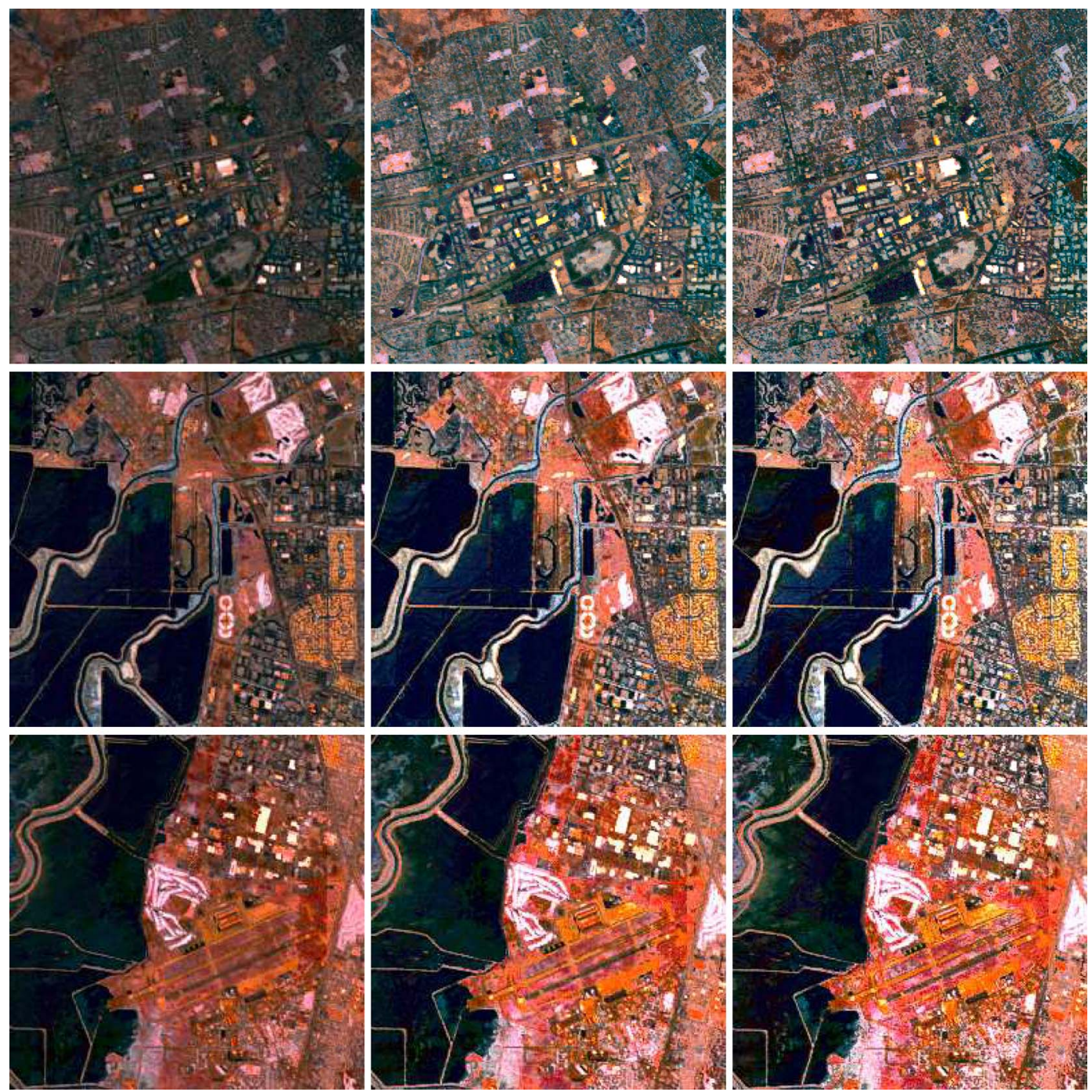

Fig. 8. Comparison of color display results obtained by our BCOCDM model with increasing values of $\gamma=0.2,0.4,0,6$ on magnified regions $(256 \times 256$ pixels) extracted from the AVIRIS images MOFFET $01-02-03$.

TABLE IV

CORRELATION $\rho$ AND CONTRAST $\delta$ FOR OUR BCOCDM ALGORITHM WiTH $\gamma=0$ AND DiFFERENT VALUES OF $N_{c}$ CORRESPONDING TO THE NUMBER OF CONNECTIONS OF OUR GRAPH (IN ADDITION TO THE Four NeAREST NeIghbors) ON THE AVIRIS IMAgES MofFET 01

\begin{tabular}{||c||c|c||}
\hline \hline$N_{c}$ & $\rho_{[\delta]}$ & CPU time in seconds \\
\hline \hline 4 & $\mathbf{0 . 9 1 8}_{[14.4]}$ & 62 \\
\hline 16 & $0.932_{[14.2]}$ & 110 \\
\hline 64 & $0.941_{[14.8]}$ & 420 \\
\hline 128 & $0.942_{[14.9]}$ & 931 \\
\hline
\end{tabular}

graphics hardware currently available on the market) and can be greatly accelerated (by a factor of 4-200) with a standard NVIDIA GPU (2004), as indicated in [33]. The source code (in $\mathrm{C}++$ language) of our algorithm BCOCDM with the set of resulting three-band color images for each hyperspectral data cube is publicly available at the following HTTP address www.iro.umontreal.ca/ mignotte/ResearchMaterial/bcocdm in order to make possible eventual comparisons with future multidimensional imagery color display algorithms and/or different performance measures.

\section{CONCLUSION}

In this paper, we have presented a new nonlinear dimensionality-reduction model for the color display of hyperspectral images. This dimensionality-reduction model is based on two complementary but contradictory criteria of good visualization, namely, accuracy and contrast with an internal parameter, allowing us to encode, for the user or for a specific application, the balance or the importance between these two criteria. This dimensionality-reduction problem was originally cast as a global nonlinear optimization problem for which an efficient optimization in several steps has also been proposed. While being simple to implement, and relatively fast, the proposed procedure allows the nonlinearity of the hyperspectral data to be considered without a preprocessing data step and performs competitively among the state-of-the-art multidimensional imagery color display methods recently proposed in the literature. Furthermore, as already noted, our method allows us, at convergence, to successively increase $\gamma$ and save the resulting color visualizations. This generation of set of color displays with increasing contrast enhancement allows us to gradually distinguish the different existing materials in this scene, 
TABLE V

METRICS $\rho$ AND $\delta$ FOR INCREASING VALUES OF $\gamma$ AND DifFERENT Sizes of $N_{s}$ FOR THE LUNLAKE 01 $\operatorname{AVIRIS~SCENE}\left(N_{s}=61\right.$ IS THE VAlue Used IN OUR APPLICATION $)$

\begin{tabular}{||c|c||c|c|c|c|c||}
\hline \hline$N_{S}$ & \multicolumn{1}{|c||}{$\gamma$} & 0.05 & 0.1 & 0.15 & 0.2 & 0.4 \\
\hline \hline 11 & $0.969_{[12.0]}$ & $0.977_{[16.3]}$ & $0.973_{[16.7]}$ & $0.965_{[18.1]}$ & $0.953_{[19.4]}$ & $0.905_{[22.3]}$ \\
\hline 31 & $0.976_{[12.5]}$ & $0.981_{[17.0]}$ & $0.973_{[18.5]}$ & $0.963_{[20.1]}$ & $0.955_{[20.8]}$ & $0.909_{[24.3]}$ \\
\hline $\mathbf{6 1}$ & $0.982_{[13.1]}$ & $0.984_{[17.6]}$ & $0.971_{[20.4]}$ & $0.966_{[21.2]}$ & $0.950_{[23.0]}$ & $0.911_{[25.9]}$ \\
\hline 91 & $0.984_{[13.1]}$ & $0.985_{[17.6]}$ & $0.972_{[20.3]}$ & $0.967_{[21.2]}$ & $0.953_{[23.0]}$ & $0.918_{[26.2]}$ \\
\hline \hline
\end{tabular}

TABLE VI

RunNing Time (In SECONDS) FOR OUR Algorithm AND AVERAGE RUNNING TIME FOR THE OTHER COLOR DisPlay MODELS

\begin{tabular}{||c||c|c|c|c||}
\hline \hline & bcocdm & $\mathrm{M}_{4}$ ICD [24] & {$[23]$} & CMF [10] \\
\hline \hline Lun.lake $_{01}$ & 62 & $\approx 280$ & $\approx 10$ & $\approx 5$ \\
\hline Lun.lake $_{02}$ & 63 & $\approx 280$ & $\approx 10$ & $\approx 5$ \\
\hline Cuprite $_{01}$ & 63 & $\approx 280$ & $\approx 10$ & $\approx 5$ \\
\hline Cuprite $_{02}$ & 63 & $\approx 280$ & $\approx 10$ & $\approx 5$ \\
\hline Moffett $_{01}$ & 73 & $\approx 280$ & $\approx 10$ & $\approx 5$ \\
\hline Moffett $_{02}$ & 62 & $\approx 280$ & $\approx 10$ & $\approx 5$ \\
\hline \hline
\end{tabular}

particularly between those that have very similar spectral signatures, thus making easier and more reliable the interpretation and quick overview of such multidimensional hyperspectral images. Nevertheless, let us recall that, in our model, the regularization parameter $\gamma$ has to be manually determined after an exhaustive search consisting in running our algorithm with a gradual increase of the parameter $\gamma$ and in accepting, as output mapping, the solution image for which an interesting compromise between the obtained score values of accuracy and contrast is finally found. An automatic estimation of this parameter, in a single run of the algorithm, to get a maximum contrast and an accuracy value that is maintained above a minimum value, or inversely, would require further research.

\section{ACKNOWLEDGMENT}

The author would like to thank the anonymous reviewers for their many valuable comments and suggestions that helped to improve both the technical content and the presentation quality of this paper.

\section{REFERENCES}

[1] H. Du, H. Qi, X. Wang, and R. Ramanath, "Band selection using independent component analysis for hyperspectral image processing," in Proc. 32nd Appl. Imagery Pattern Recog. Workshop, Washington, DC, Oct. 2003, pp. 93-99.

[2] X. Jia and J. A. Richard, "Segmented principal components transformation for efficient hyperspectral remote-sensing image display and classification," IEEE Trans. Geosci. Remote Sens., vol. 37, no. 1, pp. 538-542, Jan. 1999.

[3] P. Switzer and A. A. Green, "Min/max autocorrelation factors for multivariate spatial statistics," Stanford Univ., Stanford, CA, Tech. Rep. 6, 1984.

[4] R. Roger, "A faster way to compute the noise-adjusted principal component transform matrix," IEEE Trans. Geosci. Remote Sens., vol. 32, no. 6, pp. 1194-1196, Jun. 1994.

[5] C.-I. Chang and Q. Du, "Interference and noise adjusted principal components analysis," IEEE Trans. Geosci. Remote Sens., vol. 37, no. 9, pp. 2387-2396, Sep. 1999.
[6] L. O. Jimenez-Rodriguez, E. Arzuaga-Cruz, and M. Velez-Reyes, "Unsupervised linear featu re-extraction methods and their effects in the classification of high-dimensional data," IEEE Trans. Geosci. Remote Sens., vol. 45, no. 2, pp. 469-483, Jan. 2007.

[7] C. M. Bachmann, T. Ainsworth, and R. Fusina, "Exploiting manifold geometry in hyperspectral imagery," IEEE Trans. Geosci. Remote Sens., vol. 43, no. 3, pp. 441-454, Mar. 2005.

[8] V. Tsagaris and V. Anastassopoulos, "Multispectral image fusion for improved RGB representation based on perceptual attributes," Int. J. Remote Sens., vol. 26, no. 15, pp. 3241-3254, Aug. 2005.

[9] J. Nascimento and J. Dias, "Does independent component analysis play a role in unmixing hyperspectral data?" IEEE Trans. Geosci. Remote Sens., vol. 43, no. 1, pp. 175-187, Jan. 2005.

[10] N. P. Jacobson and M. R. Gupta, "Design goals and solutions for display of hyperspectral images," IEEE Trans. Geosci. Remote Sens., vol. 43, no. 11, pp. 2684-2692, Nov. 2005.

[11] N. P. Jacobson, M. R. Gupta, and J. Cole, "Linear fusion of image sets for display," IEEE Trans. Geosci. Remote Sens., vol. 45, no. 10, pp. $3277-$ 3288, Oct. 2007.

[12] K. Kotwal and S. Chaudhuri, "Visualization of hyperspectral images using bilateral filtering," IEEE Trans. Geosci. Remote Sens., vol. 48, no. 5, pp. 2308-2316, Apr. 2010.

[13] S. K. Rogers, T. A. Wilson, and M. Kabrisky, "Perceptual-based image fusion for hyperspectral data," IEEE Trans. Geosci. Remote Sens., vol. 35, no. 4, pp. 1007-1017, Jul. 1997.

[14] S. Mitra, H. Li, and B. Manjunath, "Multisensor image fusion using the wavelet transform," Comput. Vis. Graph. Image Process., vol. 57, no. 3, pp. 627-640, 1995.

[15] B. Demir, A. Celebi, and S. Erturk, "A low-complexity approach for the color display of hyperspectral remote-sensing images using onebit-transform-based band selection," IEEE Trans. Geosci. Remote Sens., vol. 47, no. 1, pp. 97-105, Jan. 2009.

[16] J. M. Duarte-Carvajalino, G. Sapiro, M. Velez-Reyes, and P. E. Castillo, "Multiscale representation and segmentation of hyperspectral imagery using geometric partial differential equations and algebraic multigrid method," IEEE Trans. Geosci. Remote Sens., vol. 46, no. 8, pp. 24182434, Aug. 2008.

[17] S. Cai, Q. Du, and R. J. Moorhead, "Hyperspectral imagery visualization using double layers," IEEE Trans. Geosci. Remote Sens., vol. 45, no. 10, pp. 3028-3036, Oct. 2007.

[18] S. Cai, Q. Du, and R. Moorhead, "Feature-driven multilayer visualization for remotely sensed hyperspectral imagery," IEEE Trans. Geosci. Remote Sens., vol. 48, no. 9, pp. 3471-3481, Aug. 2010.

[19] N. Renard and S. Bourennane, "Dimensionality reduction based on tensor modeling for classification methods," IEEE Trans. Geosci. Remote Sens., vol. 47, no. 4, pp. 1123-1131, Mar. 2009.

[20] A. Mohan, G. Sapiro, and E. Bosh, "Spatially coherent nonlinear dimensionality reduction and segmentation of hyperspectral images," IEEE Geosci. Remote Sens. Lett., vol. 4, no. 2, pp. 206-210, Apr. 2007.

[21] L. Zhang, Y. Zhong, B. Huang, J. Gong, and P. Li, "Dimensionality reduction based on clonal selection for hyperspectral imagery," IEEE Trans. Geosci. Remote Sens., vol. 45, no. 12, pp. 4172-4186, Nov. 2007.

[22] Q. Du, N. Raksuntorn, S. Cai, and R. Moorhead, "Color display for hyperspectral imagery," IEEE Trans. Geosci. Remote Sens., vol. 46, no. 6, pp. 1858-1866, Jun. 2008.

[23] M. Cui, A. Razddan, J. Hu, and P. Wonka, "Interactive hyperspectral image visualization using convex optimization," IEEE Trans. Geosci. Remote Sens., vol. 47, no. 6, pp. 1673-1684, Jun. 2009.

[24] M. Mignotte, "A multiresolution Markovian fusion model for the color visualization of hyperspectral images," IEEE Trans. Geosci. Remote Sens., vol. 48, no. 12, pp. 4236-4247, Dec. 2010.

[25] W. Torgerson, "Multidimensional scaling: I. Theory and method," Psychometrika, vol. 17, no. 4, pp. 401-419, Dec. 1952.

[26] T. F. Cox and M. A. A. Cox, Multidimensional Scaling. London, U.K.: Chapman \& Hall, 1994. 
[27] C. Faloutsos and K. Lin, "Fastmap: A fast algorithm for indexing, datamining and visualization," in Proc. ACM SIGMOD Int. Conf. Manage. Data (SIGMOD), May 1995, pp. 163-174, source code of FastMap available on his webpage.

[28] J. C. Platt, "Fastmap, metricmap, and landmark mds are all nystrom algorithms," in Proc. 10th Int. Workshop AISTATS, 2005, pp. 261-268.

[29] G. R. Hjaltason and H. Samet, "Properties of embedding methods for similarity searching in metric spaces," IEEE Trans. Pattern Anal. Mach. Intell., vol. 25, no. 5, pp. 530-549, May 2003.

[30] N. Metropolis, A. W. Rosenbluth, M. N. Rosenbluth, A. H. Teller, and E. Teller, "Equation of state calculations by fast computing machines," $J$. Chem. Phys., vol. 21, no. 6, pp. 1087-1092, Jun. 1953.

[31] T. M. Lillesand, R. Kiefer, and J. W. Chipman, Remote Sensing and Image Interpretation, 5th ed. Hoboken, NJ: Wiley, 2003.

[32] G. Vane, R. Green, T. Chrien, H. Enmark, E. Hansen, and W. Porter, "The Airborne Visible/Infrared Imaging Spectrometer, (AVIRIS)," Remote Sens. Environ., vol. 44, no. 2/3, pp. 127-143, May/Jun. 1993.

[33] P.-M. Jodoin and M. Mignotte, "Markovian segmentation and parameter estimation on graphics hardware," J. Electron. Imaging, vol. 15, no. 3, pp. 033 015-1-033015-15, Jul. 2006.

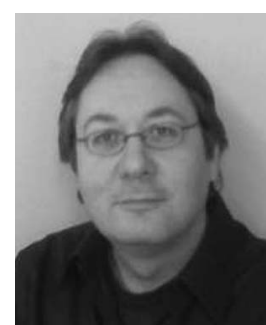

Max Mignotte received the DEA (postgraduate degree) in digital signal, image, and speech processing from the Institut National Polytechnique de Grenoble, France, Grenoble, in 1993 and the Ph.D. degree in electronics and computer engineering from the University of Bretagne Occidentale, Brest, France, and the Digital Signal Laboratory (GTS), French Naval Academy, France, in 1998.

He was an Institut National de Recherche en Informatique et en Automatique Postdoctoral Fellow with the Département d'Informatique et de Recherche Opérationnelle (DIRO), University of Montreal, Quebec, Canada, from 1998 to 1999, where he is currently with the Computer Vision and Geometric Modeling Laboratory as an Associate Professor. He is also a Member of the Laboratoire de Recherche en Imagerie et Orthopedie (LIO), Centre de Recherche du Centre Hospitalier de l'Université de Montréal (CHUM), Hopital Notre-Dame, and Researcher at CHUM. His current research interests include statistical methods, Bayesian inference, and hierarchical models for high-dimensional inverse problems. 